\title{
ANTECIPAÇÃO DO CONTROLE DA FERRUGEM-DO-CAFEEIRO (Hemileia vastatrix) COM DIFERENTES FUNGICIDAS
}

\author{
ANTICIPATION OF CONTROL OF COFFEE RUST (Hemileia vastatrix) WITH \\ DIFFERENT FUNGICIDES \&
}

\section{ANTICIPACIÓN DEL CONTROL DE LA ÓXIDA DEL CAFÉ (Hemileia vastatrix) CON DIFERENTES FUNGICIDAS \&}

Recebido em: 08/09/2021 - Aprovado em: 28/12/2021 - Publicado em: 22/11/2021

http://dx.doi.org/10.18011/bioeng2021v15n3p484-496

Hernane de Souza' (hernanedesouza@yahoo.com.br)

Kleso Silva Franco Júnior ${ }^{1}$ (kleso.junior@yahoo.com.br)

Giselle Prado Brigante ${ }^{1}$ (giselle.brigante@gmail.com)

Márcio de Souza Dias² (marciodesouzadias2013@gmail.com)

Natália Scalco Ferreira ${ }^{3}$ (nataliascalco85@gmail.com)

\footnotetext{
${ }^{1}$ Centro Superior de Ensino e Pesquisa (CESEP). Machado, MG, Brasil.

2 Secretaria de Estado de Educação de Minas Gerais. Serrania, MG, Brasil.

3 Instituto Federal do Sul de Minas (IFSULDEMINAS - Campus Machado). Machado, MG, Brasil.
}

\section{RESUMO}

O objetivo desta pesquisa foi avaliar a antecipação do controle da ferrugem do cafeeiro (Catuai Amarelo IAC 62) com diferentes fungicidas, sendo realizada em uma lavoura de aproximadamente 23 anos. O delineamento experimental foi em blocos casualizados (DBC), constituído de 5 tratamentos com 4 repetições, totalizando 20 parcelas experimentais. Os tratamentos foram: controle (água); Ciproconazol + Azoxistrobina, Piraclostrobina + Epoxiconazol, Flutriafol, Ciproconazol. Foi avaliado o nível incidência de ferrugem no ramo plagiotrópico e o crescimento de internódios aos 14, 28, 42, 56 e 70 dias após a aplicação (DAA). O controle antecipado da ferrugem com fungicidas Piraclostrobina + Epocoxiconal e Flutrifol aos 28DAA, 56DAA resultaram em menores níveis, aos 56DAA os mesmos fungicidas e também o Ciproconazol e aos 70DAA o Flutriafol, com o menor nível de ferrugem. Já em relação ao número de internódios dos ramos plagiotrópicos aos 70DAA o Flutriafol foi o que acarretou menor número.

Palavras-chave: Coffee arábica. Doenças. Pulverização. 
A produção mundial de café é de aproximadamente 143 a 152 milhões de sacas ano, tendo destaque o Brasil com o maior parque cafeeiro, de aproximadamente em 2,16 milhões de hectares (cultivados com café arábica e canephora), sendo 85,2\% destas áreas em lavouras em produção, resultando na produção de 50,9 milhões de sacas em 2019, representando por mais de $35 \%$ da produção total de café mundial (CONAB, 2019)

Para o sucesso na cafeicultura, é necessário realizar um bom manejo das lavouras, os quais envolve todo o processo produtivo, desde a escolha da semente, viveiro onde irá produzir a muda, preparo do solo para plantio, época de plantio e todos os tratos culturais. Outro detalhe importante é o monitoramento das pragas, doenças e plantas daninhas, conhecendo-os é possível ter mais facilidade e assertividade na tomada de decisões (MESQUITA, 2016).

Dentre as doenças que incidem na cultura do café a ferrugem é de maior importância por causar grandes prejuízos à cafeicultura, pode ocorrer praticamente em todas as regiões produtoras (POZZA et al., 2010) tendo como agente etiológico o fungo Hemileia vastatrix com 33 raças fisiológicas do fungo em todo o mundo, deste total, doze já foram relatadas no Brasil associadas à espécie Coffea arabica. que possui 33 raças fisiologicas distribuidas em todo mundo. Sendo um fungo biotrófico, isto é, não sobrevive em restos de culturais como saprófita (POZZA et al., 2010). Os sintomas da doença caracterizam-se por manchas arredondadas, com massa pulverulenta de esporos de cor amarelada na face inferior das folhas (Cada lesão da ferrugem pode ter centenas de milhares de esporos, (MATIELLO et al., 2019a), estes danos segundo Pozza et al. (2010), irá causar a desfolha, o que irá contribuir com perdas significativas na produção de até $50 \%$ e redução no tamanho do fruto, além de perdas de ramos laterais e menor longevidade das plantas de café.

O controle químico de doenças de plantas é uma alternativa eficiente e viável economicamente, contribuindo para obtenção de altas produtividades (KIMATI et al., 2011), sendo um dos métodos mais utilizados no Brasil para o controle da ferrugem do Cafeeiro.

$O$ uso de fungicidas pode ser preventivo e curativo, sendo que os fungicidas do grupo dos triazóis (Ciproconazol, Azoxistrobina, Piraclostrobina, Epoxiconazol, Ciproconazol e Flutriafol), apresentam boa eficiência, sendo geralmente recomendado sua aplicação no período chuvoso do ano, o qual vai de novembro a março na região sudeste (MESQUITA 
et al., 2016). O Futriafol também pode ser aplicados via solo em sistema Drench, sendo absorvido pelo sistema radicular (MATIELLO et al., 2010).

O controle químico com uso de fungicidas são eficientes para o controle de doenças de plantas, porém apresentam especificidade, o que condiciona diferenças no resultado do controle de diferentes patógenos. Os que são sistêmicos apresentam ação em sitio especifico do fungo, alterando por exemplo a síntese de proteínas, divisão celular, transporte de elétron e etc. sendo fundamental uma recomendação técnica por profissional que conheça sobre a cultura, fitossanidade, tecnologia de aplicação e especialmente sobre fungicidas (GARCIA, 1999).

Grigolli (2015), relata que a antecipação do controle de doenças fúngicas em soja é uma alternativa interessante para manejo, reduzindo significativa a incidência de algumas doenças. Assim sendo, para a cultura do cafeeiro a antecipação do controle da ferrugem pode ser uma opção para que a principal doença a "ferrugem" não tenha níveis elevados de incidência na época de maior incidência.

O objetivo desta pesquisa foi avaliar a antecipação do controle da ferrugem do cafeeiro (Hemileia vastatrix) com diferentes fungicidas em lavoura de Catuai Amarelo (IAC $62)$.

\section{MATERIAL E MÉTODOS}

A pesquisa foi realizada na Fazenda Sertãozinho Ltda., no bairro Cachoeirinha, no município de Campestre, Sul de Minas - Brasil, (21ํ 38' 53,69' S, 46 19' 18,44' W e altitude 1045,45 metros), no período de julho a outubro de 2019, visando um tratamento antecipado para reduzir os esporos e não ter explosão da doença na época mais tradicional de sua incidência, em uma lavoura de café arábica, variedade Catuaí Amarelo (IAC 62), com espaçamento de $3,50 \times 0,70$ metros, estande de plantas de 4.081 plantas ha-1, com 23 anos, cujos dados de produtividade dos últimos anos podem ser observados na Tabela 1, onde após a colheita de 2018 foi realizada a poda do tipo esqueletamento na lavoura, consequentemente em 2019 a lavoura não teve produção. 
Tabela 1. Produtividade da Lavoura nos últimos 6 anos.

\begin{tabular}{lc}
\hline Ano & Produtividade $\left(\right.$ sacas ha $\left.{ }^{-1}\right)$ \\
\hline 2014 & 42 \\
2015 & 20 \\
2016 & 68 \\
2017 & 26 \\
2018 & 53 \\
2019 & 0 * \\
\hline Média & 34,83 \\
\hline
\end{tabular}

*Lavoura foi esqueletada após colheita de 2018, encontra-se em safra zero em 2019

O delineamento experimental adotado foi o de blocos ao acaso (DBC), constituído de 5 tratamentos (Tabela 2) com 4 repetições, totalizando 20 parcelas experimentais, onde os quatros fungicidas foram aplicados utilizando-se a maior dosagem de registro conforme bula. $O$ volume de calda foi o mesmo para todos os tratamentos (fungicidas) $400 \mathrm{~L} \mathrm{ha}^{-1}$, 0 que correspondeu em $9,801 \mathrm{~L}^{-1}$ por parcela.

Tabela 2. Tratamentos utilizados

\begin{tabular}{|c|c|c|c|}
\hline Tratamentos & Princípios ativos e i.a. & $\begin{array}{l}\text { Dosagem } \\
\left(\mathrm{mL} \mathrm{ha}^{-1}\right)\end{array}$ & $\begin{array}{l}\text { Espalhante } \\
\text { adesivo } \\
\text { Break Thru } \\
\left(\mathrm{mL} \mathrm{ha}^{-1}\right)\end{array}$ \\
\hline Controle & Água & - & - \\
\hline $\begin{array}{l}\text { Piraclostrobina + } \\
\text { Epoxiconazol }\end{array}$ & $\begin{array}{l}\text { Piraclostrobina }(260 \mathrm{~g} / \mathrm{L} \text { de i.a })+ \\
\text { Epoxiconazol }(160 \mathrm{~g} / \mathrm{L} \text { de i.a. })\end{array}$ & 450 & 100 \\
\hline Ciproconazol & $\begin{array}{l}\text { Ciproconazol ( } 80 \mathrm{~g} / \mathrm{L} \text { de i.a })+ \\
\text { Azoxistrobina ( } 200 \mathrm{~g} / \mathrm{L} \text { de i.a.) }\end{array}$ & 750 & 100 \\
\hline Flutriafol & Flutriafol (125 g/L de i.a.) & 2000 & 100 \\
\hline Ciproconazol & Ciproconazol (100 g/L de i.a.) & 750 & 100 \\
\hline
\end{tabular}

Em todos os tratamentos "fungicidas", foram adicionados ao espalhante adesivo do grupo dos siliconados, não-iônico (Break Thru), a 0,1\% do volume de calda, ou seja 9,80 $\mathrm{mL}$. 
As parcelas foram constituídas de $49 \mathrm{~m}^{2}$ na linha do cafeeiro, contendo 20 plantas que receberam os tratamentos. Foram avaliadas as 12 plantas centrais em cada parcela, a distância entre as parcelas foi de 40 plantas, as quais não receberam nenhum tratamento.

Antes da aplicação dos tratamentos, realizou-se o monitoramento da ferrugem, onde os níveis encontravam na média de $25 \%$ de incidência (MATIELLO, 2019b). As aplicações dos fungicidas foram feitas em 03/08/2019, de forma mecânica com trator e tanque atomizador de 400 litros, com bicos pontas Jacto Cônico 11002, utilizando um volume de calda equivalente a 400 litros de calda por hectare, e vazão de $775 \mathrm{~mL}$ por minuto por ponta.

Os dados metrológicos do município de Campestre, durante o período de condução da pesquisa estão apresentados na Tabela 3, sendo coletados na base de dados da Estação Meteorológica da Cooxupé.

Tabela 3. Dados meteorológicos de Campestre - MG na Estação Cooxupé calculado de acordo com a metodologia de Thorntwaite \& Mather (1955) e evapotranspiração segundo Camargo (1971).

\begin{tabular}{llllllll}
\hline $\begin{array}{l}\text { Meses } \\
(\mathbf{2 0 1 9})\end{array}$ & Temp $\left({ }^{\circ} \mathbf{C}\right)$ & $\begin{array}{l}\text { Pluviosidade } \\
(\mathbf{m m})\end{array}$ & ETP $(\mathbf{m m})$ & $\begin{array}{l}\text { ETR } \\
(\mathbf{m m})\end{array}$ & ARM $(\mathbf{m m})$ & DEF $(\mathbf{m m})$ & $\begin{array}{l}\text { EXC } \\
(\mathbf{m m})\end{array}$ \\
\hline Jan & 23,4 & 166,2 & 125,6 & 125,6 & 100,0 & 0,0 & 40,6 \\
Fev & 22,3 & 269,6 & 105,8 & 105,8 & 100,0 & 0,0 & 163,8 \\
Mar & 21,9 & 200,0 & 106,1 & 106,1 & 100,0 & 0,0 & 93,9 \\
Abr & 21,4 & 180,8 & 87,4 & 87,4 & 100,0 & 0,0 & 93,4 \\
Mai & 19,2 & 98,6 & 67,9 & 67,9 & 100,0 & 0,0 & 27,7 \\
Jun & 17,2 & 5,0 & 51,1 & 41,9 & 63,1 & 9,1 & 0,0 \\
Jul & 16,0 & 28,0 & 47,4 & 39,1 & 52,0 & 9,3 & 0,0 \\
Ago & 17,6 & 25,0 & 55,3 & 38,6 & 38,4 & 16,7 & 0,0 \\
Set & 22,0 & 113,6 & 99,9 & 99,9 & 52,00 & 0,0 & 0,0 \\
Out & 22,6 & 31,4 & 105,2 & 58,6 & 24,9 & 46,7 & 0,0 \\
Nov & 22,2 & 170,4 & 109,8 & 109,8 & 85,5 & 0,0 & 0,0 \\
\hline
\end{tabular}

Fonte: http://sismet.cooxupe.com.br:9000/bh/estacoes/mensal/

As avaliações do nível de incidência ou ausência de ferrugem-do-cafeeiro segundo metodologia de Matiello (2019b), sendo a primeira avaliação realizada aos 14 dias após a aplicação (DAA), as demais avaliações ocorreram a cada 14 dias sendo a última avaliação aos 70 DAA e o crescimento dos internódios foram realizados de acordo com a metodologia descrita por Alfonsi (2008), aos 70 DAA.

Os dados obtidos com as avaliações foram submetidos a análise estatística através do software SISVAR ${ }^{\circledR}$ (Ferreira, 2014), sendo a diferença significativa entre os tratamentos 
determinada pelo teste $\mathrm{F}<0,05$, com as médias comparadas pelo teste de Scott-Knott ao nível de $5 \%$ de probabilidade.

\section{RESULTADOS E DISCUSSÃO}

Após a aplicação dos fungicidas, a avaliação foi realizada 14, 28, 42, 56 e 70DAA, cujos valores médios das avaliações estão descritos na Tabela 4.

Tabela 4. Média das avaliações de números de folhas do cafeeiro com ferrugem (Hemileia vastratrix) aos 14, 28, 42, 56 e 70 dias após a aplicação (DAA) dos fungicidas.

\begin{tabular}{lccccccc}
\hline \multirow{2}{*}{ Tratamentos } & \multicolumn{7}{c}{ DAA } \\
\cline { 2 - 7 } & 14 & 28 & 42 & 56 & 70 & Média \\
\hline Controle & $4,8 \mathrm{~A}$ & $4,4 \mathrm{~A}$ & $4,7 \mathrm{~B}$ & $5,4 \mathrm{~B}$ & $6,0 \mathrm{~B}$ & $5,1 \mathrm{~B}$ \\
Piraclostrobina+ Epoxiconazol & $4,5 \mathrm{~A}$ & $3,0 \mathrm{~B}$ & $3,2 \mathrm{C}$ & $3,8 \mathrm{C}$ & $4,0 \mathrm{C}$ & $3,7 \mathrm{D}$ \\
Ciproconazol+ Azoxistrobina & $5,0 \mathrm{~A}$ & $5,3 \mathrm{~A}$ & $6,4 \mathrm{~A}$ & $7,5 \mathrm{~A}$ & $8,8 \mathrm{~A}$ & $6,6 \mathrm{~A}$ \\
Flutriafol & $5,5 \mathrm{~A}$ & $3,2 \mathrm{~B}$ & $3,2 \mathrm{C}$ & $3,2 \mathrm{C}$ & $3,4 \mathrm{D}$ & 3,7 D \\
Ciproconazol & $5,0 \mathrm{~A}$ & $2,8 \mathrm{~B}$ & $4,7 \mathrm{~B}$ & $4,0 \mathrm{C}$ & $4,3 \mathrm{C}$ & 4,2 C
\end{tabular}

${ }^{\star}$ Médias seguidas das mesmas letras na coluna não diferem estatisticamente entre si pelo teste de ScottKnott a $5 \%$ de probabilidade.

Os resultados obtidos e submetidos à análise estatística, para a incidência ou não de ferrugem nas folhas do café, demonstraram que aos 14DAA dos fungicidas, os fungicidas e o controle não obtiveram diferença estatística.

Aos 28DAA, os fungicidas que apresentaram melhores resultados foram nos tratamentos com Ciproconazol (100 g L-1), Piraclostrobina (260 g L-1) + Epoxiconazol (160 $\mathrm{g} \mathrm{L}^{-1}$ ), e o Flutriafol (125 $\mathrm{g} \mathrm{L}^{-1}$ ), aos 42DAA, foram nos tratamentos que receberam Piraclostrobina (260 $\left.\mathrm{g} \mathrm{L}^{-1}\right)+$ Epoxiconazol $\left(160 \mathrm{~g} \mathrm{~L}^{-1}\right)$, e Flutriafol $\left(125 \mathrm{~g} \mathrm{~L}^{-1}\right)$ e aos $56 \mathrm{DAA}$, os melhores resultados foram os tratamentos Flutriafol $\left(125 \mathrm{~g} \mathrm{~L}^{-1}\right)$, Piraclostrobina (260 $\mathrm{g} \mathrm{L}^{-}$ 1) + Epoxiconazol (160 $\left.\mathrm{g} \mathrm{L}^{-1}\right)$, com Ciproconazol (100 $\left.\mathrm{g} \mathrm{L}^{-1}\right)$.

Durante as avaliações, houve diferenças entre a eficiência dos fungicidas de acordo com seus mecanismos de ação sobre a Hemileia vastatrix, se destacando aos 56 DAA o Flutriafol (125 $\left.\mathrm{g} \mathrm{L}^{-1}\right)$, Piraclostrobina $\left(260 \mathrm{~g} \mathrm{~L}^{-1}\right)$ + Epoxiconazol (160 $\left.\mathrm{g} \mathrm{L}^{-1}\right)$, com Ciproconazol (100 $\left.\mathrm{g} \mathrm{L}^{-1}\right)$. 
Matos et al. (2016) trabalhando com a cultivar Catuaí Vermelho (IAC 144) a curva de progresso de incidência da doença (CPID) da ferrugem demonstrou que os produtos Azoxistrobina + Ciproconazol + Tebuconazol, Azoxistrobina + Ciproconazol - 500 e $750 \mathrm{~mL}$ ha $^{-1}$ e Piraclostrobina + Epoxiconazol apresentaram bom controle da doença, diferentemente do Tebuconazol aplicado isoladamente. Resultados aproximados com o deste trabalho nas avaliações aos 28,42 e 56DAA.

Aos 70DAA, o fungicida que apresentou o melhor resultado foi o tratamento que recebeu Flutriafol (125 $\mathrm{g} \mathrm{L}^{-1}$ ). Esse resultado corrobora com trabalhos realizados por Matiello et al. (2017), onde o controle permitiu que a incidência de ferrugem não fosse superior a $31,5 \%$. Esse fato explica por que não se deve aguardar índices altos de incidência de ferrugem em anos de alta carga e em condições de clima favorável à doença, para que seja iniciado o controle com fungicidas sistêmicos.

Corroborando com as observações do trabalho de Alfonsi et al. (2019) onde os mesmos relataram que é importante evitar o atraso do início do controle da ferrugem para evitarmos a severidade do ataque da doença.

Ainda aos 70 dias, os tratamentos com Flutriafol, se mostrou com os menores níveis de incidência de ferrugem diferindo dos demais, segundo pelo Piraclostrobina + Epocoxiconazol + Ciproconazol, não diferiram estatisticamente entre si, sendo superiores ao tratamento controle, e o tratamento com Ciproconazole + Azoxistrobina, apresentou incidência maior de ferrugem do que o controle que não havia recebido nenhum fungicida.

Quanto ao número de internódios desenvolvidos aos 70DAA dos fungicidas, os resultados encontram-se na Tabela 5.

Tabela 5. Média das avaliações de números de internódios do cafeeiro aos 70 dias após a aplicação (DAA) dos fungicidas

\begin{tabular}{lc}
\hline Tratamentos & Número de internódios \\
\hline 1- Controle & $2,00 \mathrm{~A}$ \\
2- Piraclostrobina + Epoxiconazol & $1,60 \mathrm{~A}$ \\
3- Ciproconazol+ Azoxistrobina & $1,80 \mathrm{~A}$ \\
4- Flutriafol & $1,00 \mathrm{~B}$ \\
5- Ciproconazol & $1,80 \mathrm{~A}$ \\
\hline
\end{tabular}

${ }^{*}$ Médias seguidas das mesmas letras na coluna não diferem estatisticamente entre si pelo teste de ScottKnott a $5 \%$ de probabilidade. 
Conforme observa-se na Tabela 5, houve uma diferença estatística entre os tratamentos com relação ao número de internódios desenvolvidos, onde o fungicida Flutriafol (125 $\mathrm{g} \mathrm{L}^{-1}$ ), apresentou menor crescimento dos ramos plagiotrópicos no período de 70DAA evidenciando que alguns fungicidas exercem redução de crescimento da planta. Os demais fungicidas apresentaram crescimento de internódios similares estatisticamente ao tratamento controle de fungicida. Em relação aos fungicidas, exceto o Flutriafol, os demais apresentaram crescimento de internódios similares estatisticamente ao tratamento controle.

Pesquisa realizada por Sandy et al. (2011), contribui com os obtidos neste estudo, não se observando efeitos positivos da aplicação de diferentes fontes de estrobilurinas via Drench ou via foliar, em relação ao número de ramos plagiotrópicos e altura de plantas de cafeeiro da variedade Catuaí (IAC 62).

Os fungicidas do grupo dos triazois ( Ciproconazol, Epoxiconal e Flutriafol) atuam como inibidores da biossíntese de Ergosterol, inibindo da biossíntese de esterol (G1), agindo no sitio alvo, inibindo a demetilação do C14,(FARC), sendo sistêmicos, absorvidos e translocados pela planta de forma acropetal, podendo atuar tanto como protetores ou curativos, tendo ação tóxica sobre a germinação de esporos, o que pode ser considerado pelos resultados obtidos, proporcionando ao futriafol este efeito em antecipação no controle da ferrugem do cafeeiro (EHR \& KEMMITT, 2002). Já os fungicidas do grupo da estrubirulinas (piraclostrobina e azoxystrobina), atuam na sobre a respiração de manutenção pode aumentar a taxa de fotossíntese líquida da planta (FARGAN et al., 2010) podendo assim afetar o crescimento de ramos plagiotrópicos, porém no trabalho em questão não foi evidenciado este efeito, além deste efeito estes fungicidas atuam preventivamente inibindo a germinação de esporos, o que pode ser observado no resultado deste trabalho.

O efeito da estrobilurina piraclostrobina foi considerado como rápido, em torno de 3 horas após da primeira aplicação, onde a taxa fotossintética das plantas tratadas com a estrobilurina piraclostrobina, apresentou um crescimento de $3 \%$ e $10 \%$. Os resultados indicaram que a estrobilurina piraclostrobina afeta a taxa de assimilação de carbono e de nitrogênio com aumento na produtividade.

Os fungicidas do grupo dos triazois (Ciproconazol, Epoxiconal e Flutriafol) atuam como inibidores da biossíntese de Ergosterol, inibindo da biossíntese de esterol (G1), agindo no sitio alvo, inibindo a demetilação do C14,(FARC), sendo sistêmicos, absorvidos 
e translocados pela planta de forma acropetal, podendo atuar tanto como protetores ou curativos, tendo ação tóxica sobre a germinação de esporos, o que pode ser considerado pelos resultados obtidos, proporcionando ao Flutriafol este efeito em antecipação no controle da ferrugem do cafeeiro (EHR e KEMMITT, 2002).

Os fungicidas do grupo da estrubirulinas (piraclostrobina e azoxystrobina), atuam sobre a respiração de manutenção podendo aumentar a taxa de fotossíntese líquida da planta (FARGAN et al., 2010) podendo assim afetar o crescimento de ramos plagiotrópicos, porém no trabalho em questão não foi evidenciado este efeito, além disso, estes fungicidas atuam preventivamente inibindo a germinação de esporos, o que pode ser observado no resultado deste trabalho.

Ainda avaliando a média em relação aos períodos avaliados, ficou evidente que o Futriafol e o Piraclostrobina+ Epoxiconaloze, se destacaram quando comparados aos demais fungicidas.

\section{CONCLUSÕES}

Conclui-se que a antecipação do controle da ferrugem do cafeeiro com o uso do fungicida Flutriafol via foliar foi eficiente na avaliação aos 70 DAA, e com os fungicidas Flutriafol e o Piraclostrobina + Epoxiconazole entre a média das avaliações no período, assim permitindo uma estratégia de manejo para o controle da doença.

\section{REFERÊNCIAS}

ALFONSI, W.M.V.; COLTRI, P.P.; ZULLO JÚNIOR, J.; PATRÍCIO, F.R.A.; ALFONSI, E.L. Período de incubação da ferrugem do cafeeiro. Summa Phytopathologica, 45(2), 134140, 2019. Epub July 10, 2019. Disponível em: <https://doi.org/10.1590/01005405/187216>. Acesso em: 21 de abril de 2019.

ALFONSI, E.L. Uso de índices fenológicos em modelos de previsão de produtividade do cafeeiro. Piracicaba: ESALQ/USP, 2008. 104p. Tese Doutorado.

ALMEIDA, S.R.; MATIELLO, J.B.; FERREIRA, R.A. Efeito de dose, número de aplicações e adição de cobre ou espalhante na ação do fungicida Opus (Epoxiconazole) no controle curativo da ferrugem do cafeeiro. In: CONGRESSO BRASILEIRO DE PESQUISAS CAFEEIRAS, 24., 1998, Rio de Janeiro. Resumos... Rio de Janeiro: PROCAFÉ, 1998. p. 49-51. 
CAMARGO, A.P. Balanço hídrico no Estado de São Paulo. 3.ed. Campinas: Instituto Agronômico, 1971. 24p. (Boletim, 116).

COMPANHIA NACIONAL DE ABASTECIMENTO (Conab). Acompanhamento da safra brasileira de café: safra 2019: segundo levantamento, maio 2019.Brasília, v.5 n.2 p.1-61. 2019. Disponível em: < https://www.conab.gov.br/component/k2/item/download/26519_d3 bb5963ecc22391abd34b0824a87a55>. Acesso em: 05 de julho de 2019.

CUNHA, R.L.; MENDES, A.N.G.; CHALFOUN, S.M. Controle químico da ferrugem do cafeeiro (Coffea arabica L.) e seus efeitos na produção e preservação do enfolhamento. Ciência e Agrotecnologia. vol.28 no.5 Lavras Set./Out. 2004. Disponível em: <http://www.scielo.br/scielo.php?script=sci_arttext\&pid=S1413-70542004000500004>. Acesso em: 14 de dezembro de 2019.

EHR, R.J.; KEMMITT, G. Periodic table of the fungicides. Indianapolis: Dow Agrosciences. Vol. 1, 2002. 18p.

FAGAN, E.B.; NETO, D.D.; VIVIAN, R.; FRANCO, R.B.; YEDA, M.P.; MASSIGNAM, L.F.; OLIVEIRA, R.F.; MARTINS, K.V. Efeito da aplicação de piraclostrobina na taxa fotossintética, respiração, atividade da enzima nitrato redutase e produtividade de grãos de soja. Bragantia, Piracicaba. v. 69, n.4, p. 771-777. 2010. Disponível em: <http://www.scielo.br/scielo.php?pid=S0006-87052010000400001\&script=sci_arttext>. Acesso em: 17 de novembro de 2019.

FERREIRA, D.F. Sisvar: um guia dos seus procedimentos de comparações múltiplas Bootstrap. Ciência e Agrotecnologia, v. 38, n. 2, p. 109-112, 2014.

GARCIA, A. Fungicidas I: utilização no controle químico de doenças e sua ação contra os fitopatógenos. Porto Velho: EMBRAPA-CPAF Rondônia, 1999. 32p. (EMBRAPA-CPAF Rondônia. Documentos, 46).

GRIGOLLI, J.F.J, Manejo de Doenças na Cultura da Soja, 2015. GRIGOLLI, J.F.J. Manejo de Doenças na Cultura da Soja. In: LOURENÇÃO, A.L.F.; GRIGOLLI, J.F.J.; MELOTTO, A.M.; PITOL, C.; GITTI, D.C.; ROSCOE, R.. (Org.). Tecnologia e Produção Soja 2013/2014. 1ed.Curitiba: Midiograf, 2014, v. 1, p. 205-231. Disponível em:

<https://www.

fundacaoms.org.br/base/www/fundacaoms.org.br/media/attachments/216/216/newarchive216.pdf>. Acesso em: 26 de Out. 2021.

KIMATI, H. et al. Manual de Fitopatologia. 4. ed. São Paulo: USP/ESALQ, 2011. 343pg.

MATIELLO, J.B.; SANTINATO, R.; GARCIA, A.W.R.; ALMEIDA, S.R.; FERNANDES, D.R. Cultura do café no Brasil: manual de recomendações. Rio de Janeiro :MAPA/Procafé; Varginha: Fundação Procafé, 2010. 546pg.

MATIELLO, J. B.; Avaliação da ferrugem do cafeeiro - diferenças entre a prática e a academia. Revista Attalea Agronegocios: v.1, n.1, Fevereiro, 2019a. Disponível em:< cafepoint.com.br/notícias/tecnicas-de-producao/avaliacao-da-ferrugem-do-cafeeiro-diferen cas-entre-a-pratica-e-a-academia-212307/>. Acesso em: 17 de agosto de 2019. 
MATIELLO, J. B.; Almeida, S. R.; Paiva, R. N.; Procafé: Avaliação da ferrugem do cafeeiro - diferenças entre prática e a academia. Notícias agrícolas, 30 de janeiro de 2019b.

Disponível em: <https://www.noticiasagricolas.com.br/noticias/cafe/229326-procafeavaliacao-da-ferrugem-do-cafeeiro-diferencas-entre-a-pratica-e-a-academia.html\#.XTgx DPJKJIU>. Acesso em: 10 de julho de 2019.

MATIELLO et al. Dose e parcelamento do fungicida flutriafol no controle da ferrugem do cafeeiro. 43 Congresso brasileiro de pesquisas cafeeiras. Poços de Caldas, MG. Fundação Procafé. 1-2 pg. 2017. Disponível em: <http://www.sbicafe.ufv.br/bitstream/han dle/123456789/9328/39_43-CBPC-2017.pdf?sequence=1\&isAllowed=y. Acesso em 27 de Out. 2021.

MATOS, G.A.; SOUSA, F.A.; JÚNIOR, J.P.; LIMA, L.M. Avaliação da mistura de fungicidas no controle de doenças do cafeeiro. Revista Getec, v.5, n.9, p.90-103. 2016. Disponível em: <http://www.fucamp.edu.br/editora/index.php/getec/article/view/756>. Acesso em: 14 de dezembro de 2019.

MESQUITA, C.M.; REZENDE, J.E.; CARVALHO, J.S; JÚNIOR, M.A.F.; MORAES, C.N.; DIAS, P.T.; CARVALO, R,M.; ARAÚJO, W.G. Manual do Café: Distúrbios Fisiológicos, Pragas e Doenças do Cafeeiro (Coffea arábica L.). EMATER, Belo Horizonte: v.1, n.1, 64p., 2016. Disponível em: < http://www.sapc.embrapa.br/arquivos/consorcio/publicacoes tecnicas/livro_disturbios_fisiologicos_pragas_doen\%C3\%A7as.pdf>. Acesso em: $21 \mathrm{de}$ abril de 2019.

POZZA, E. A.; CARVALHO, V. L.; CHALFOUN, S. M. Sintomas de injúrias causadas por doenças em cafeeiro. In: GUIMARÃES, R. J.; MENDES, A. N. G.; BALIZA, D. P. (Ed.). Semiologia do cafeeiro: sintomas de desordens nutricionais, fitossanitárias e fisiológicas. Lavras: Editora da UFLA, 2010. p. 69-101.

SANDY, E.C.; SARTORI, R.H.; NETO, B.F. Aplicação de estrobilurina piraclostrobina via drench e foliar na cultura do café. Sbicafe/UFV. 1-2p. 2011.

THORNTHWAITE, C.W.; MATHER, J.R. The water balance. Centerton, NJ: Drexel Institute of Technology - Laboratory of Climatology, v.8, n.1, 104p., 1955.

ZAMBOLIM, L.; VALE, F.X.R.; COSTA, H.; PEREIRA, A.A.; CHAVES, G.M. Epidemiologia e controle integrado da ferrugem-do-cafeeiro. In: ZAMBOLIM, L. (Ed.). O estado da arte de tecnologias na produção de café. Viçosa: UFV, 2002. cap. 10, p. 369-450. 


\begin{abstract}
The objective of this research was to evaluate the anticipation of the control of coffee rust (Catuai Amarelo IAC 62) with different fungicides, being carried out in a field of approximately 23 years. The experimental design was in randomized blocks (DBC), consisting of 5 treatments with 4 replications, totaling 20 experimental plots. The treatments were: control (water); Cyproconazole + Azoxystrobin, Pyrclostrobin + Epoxiconazole, Flutriafol, Cyproconazole. The level of rust incidence in the plagiotropic branch and the growth of internodes at 14, 28, 42, 56 and 70 days after application (DAA) were evaluated. The early control of rust with fungicides Pyraclostrobin + Epocoxiconal and Flutrifol at 28DAA, 56DAA resulted in lower levels, at 56DAA the same fungicides and also Ciproconazole and at 70DAA Flutriafol, with the lowest level of rust. Regarding the number of internodes in the plagiotropic branches at 70DAA, Flutriafol was the one that caused the lowest number.
\end{abstract}

Keywords: Coffee arabica. Diseases. Spraying.

\title{
RESUMEN
}

El objetivo de esta investigación fue evaluar la anticipación del control de la roya del café (Catuai Amarelo IAC 62) con diferentes fungicidas, llevándose a cabo en un cultivo de aproximadamente 23 años. El diseño experimental fue en bloques al azar (DBC), consistente en 5 tratamientos con 4 repeticiones, totalizando 20 parcelas experimentales. Los tratamientos fueron: control (agua); Ciproconazol + Azoxistrobina, Pirclostrobina + Epoxiconazol, Flutriafol, Ciproconazol. Se evaluó el nivel de incidencia de roya en la rama plagiotrópica y el crecimiento de entrenudos a los 14, 28, 42, 56 y 70 días después de la aplicación (DAA). El control temprano de la roya con fungicidas Pyrclostrobin + Epocoxiconal y Futrifol a 28DAA, 56DAA resultó en niveles más bajos, a 56DAA los mismos fungicidas y también Ciproconazol y a 70DAA Flutriafol, con el nivel más bajo de roya. En cuanto al número de entrenudos en las ramas plagiotrópicas a 70DAA, Flutriafol fue el que provocó el menor número.

Palabras-clave: Coffea arabica. Enfermedade. Pulverización. 


\section{LICENÇA DE USO}

Este é um artigo publicado em acesso aberto (Open Access) sob a licença Creative Commons Atribuição 4.0 Internacional (CC BY 4.0), que permite uso, distribuição e reprodução em qualquer meio, desde que o trabalho original seja corretamente citado. Mais informações em: http://creativecommons.org/licenses/by/4.0

\section{CONFLITO DE INTERESSES}

Os autores declaram que não há conflito de interesses neste trabalho.

\section{CONTRIBUIÇÕES AUTORAIS}

Autor 1: Autor do trabalho.

Autor 2: Autor do trabalho.

Autor 3: Autor do trabalho.

Autor 4: Revisor do trabalho.

Autor 5: Revisor do trabalho.

\section{FINANCIAMENTO}

O presente trabalho não contou com apoio financeiro.

\section{AGRADECIMENTO}

A Coordenação de Aperfeiçoamento de Pessoal de Nível Superior (CAPES) - Código Financeiro 001. A Fundação de Amparo à Pesquisa do Estado de Minas Gerais (FAPEMIG).

\section{COMO REFERENCIAR}

SOUZA, Hernane de et al. Antecipação do controle da ferrugem-do-cafeeiro (Hemileia vastatrix) com diferentes fungicidas. Revista Brasileira de Engenharia de Biossistemas (Tupã), v. 15, n. 3, p. 484-496, 2021. DOI:

http://dx.doi.org/10.18011/bioeng2021v15n3p484-496.

\section{RESPONSABILIBADE EDITORIAL}

Prof. Dr. Fernando Ferrari Putti ${ }^{1}$, Prof. Dr. Paulo Sérgio Barbosa dos Santos ${ }^{1}$, Prof. Dr. Eduardo Festozo Vicente ${ }^{1}$ e Prof. Dr. Diogo de Lucca Sartori ${ }^{1}$

${ }^{1}$ Universidade Estadual Paulista "Júlio de Mesquita Filho", FCE - Faculdade de Ciências e Engenharia, Tupã, SP, Brasil. 